\title{
A combined medication safety assessment of rivaroxaban with Tyrosine Kinase Inhibitors for cancer patients: focusing on CYP2J2 and CYP3A4
}

\author{
tingting Zhao ${ }^{1}$, Xuening Li $^{1}$, Yanwei Chen ${ }^{2}$, Dalong Wang ${ }^{1}$, Liyan Wang ${ }^{2}$, Shan Zhao ${ }^{3}$, \\ Changyuan Wang ${ }^{4}$, Qiang Meng ${ }^{1}$, Huijun Sun ${ }^{1}$, Kexin $\mathrm{Liu}^{1}$, and Jingjing $\mathrm{Wu}^{1}$ \\ ${ }^{1}$ Dalian Medical University \\ ${ }^{2}$ First Affiliated Hospital of Dalian Medical University \\ ${ }^{3}$ Chinese Academy of Sciences \\ ${ }^{4}$ Dalian Med Univ
}

June 30, 2021

\begin{abstract}
Background and purpose: Cancer patients are always complicated with vein thromboembolism, thus the combination of anticoagulants with anti-cancer drugs has profound foundations. This study aimed to assess the safety of rivaroxaban comminating with three tyrosine kinase inhibitors (TKIs) in cancer patients. Experimental Approach: The inhibition of three TKIs on CYP2J2- and CYP3A4-mediated rivaroxaban metabolism was first screened and then reversible and mechanism-dependent inhibitory kinetic constants were determined. Molecular docking was conducted to reveal the interactions between TKIs and CYP2J2 and CYP3A4. Finally, pharmacokinetic parameters of cancer patients were used to assess the safety. Key Results: Imatinib and gefitinib significantly reversibly inhibited CYP2J2- and CYP3A4-mediated rivaroxaban metabolism, while sunitinib only showed reversible inhibition of CYP3A4, not CYP2J2. Three TKIs also showed time-dependent inactivation of CYP3A4. Notably, sunitinib had the strongest inactivation effect on CYP3A4 than the other TKIs with a 4.00-fold IC50 shift, however, a slight effect on CYP2J2. Docking simulations revealed the relation of inhibitory activity to ChemScore. Additionally, drugdrug interaction risks of combinations were assessed using pharmacokinetic data of cancer patients. Imatinib, which showed the strongest inhibition, was predicted to cause a $114-244 \%$ increase in rivaroxaban exposure. Conclusion and Implications: Imatinib was predicted to have a moderate DDI risk when was combined with rivaroxaban. These results provide evidence for medication guidance when combining rivaroxaban with TKIs for cancer patients, and also give new insight for the DDI assessment involving rivaroxaban.
\end{abstract}

\section{A combined medication safety assessment of rivaroxaban with Tyrosine Kinase Inhibitors for cancer patients: focusing on CYP2J2 and CYP3A4}

Tingting Zhao ${ }^{\mathrm{a}, \mathrm{b} \#}$, Xuening $\mathrm{Li}^{\mathrm{a}} \mathrm{b}$, \#, Yanwei Chen ${ }^{\mathrm{c}, \#}$, Dalong Wang ${ }^{\mathrm{a}}$, Liyan Wang ${ }^{\mathrm{c}}$, Shan Zhao ${ }^{\mathrm{d}}$, Changyuan Wanga, b, Qiang Menga, b, Huijun Sun ${ }^{\mathrm{a}, \mathrm{b}}$, Kexin Liu ${ }^{\mathrm{a}}$, b, Jingjing Wu ${ }^{\mathrm{a}}$, b*

${ }^{a}$ Department of Clinical Pharmacology, College of Pharmacy, Dalian Medical University, Dalian 116044, China.

${ }^{b}$ Provincial Key Laboratory for Pharmacokinetics and Transport, Liaoning Dalian Medical University, Dalian, Liaoning, China.

${ }^{\mathrm{c}}$ Department of Pharmacy, the First Affiliated Hospital of Dalian Medical University, Dalian 116011, China.

${ }^{\mathrm{d}}$ Dalian Institute of Chemical Physics, Chinese Academy of Sciences, Dalian 116023, China. 
\# These authors contributed equally to this work.

* Correspondence:

Dr. Jingjing $\mathrm{Wu}$

ORCID: 0000-0002-5046-9996

Department of Clinical Pharmacology, College of pharmacy

Dalian Medical University

9 West Section, Lvshun South Road, Lvshunkou District, Dalian 116044, China

\begin{abstract}
Background and purpose: Cancer patients are always complicated with vein thromboembolism, thus the combination of anticoagulants with anti-cancer drugs has profound foundations. This study aimed to assess the safety of rivaroxaban comminating with three tyrosine kinase inhibitors (TKIs) in cancer patients.
\end{abstract}

Experimental Approach: The inhibition of three TKIs on CYP2J2- and CYP3A4-mediated rivaroxaban metabolism was first screened and then reversible and mechanism-dependent inhibitory kinetic constants were determined. Molecular docking was conducted to reveal the interactions between TKIs and CYP2J2 and CYP3A4. Finally, pharmacokinetic parameters of cancer patients were used to assess the safety.

Key Results: Imatinib and gefitinib significantly reversibly inhibited CYP2J2- and CYP3A4-mediated rivaroxaban metabolism, while sunitinib only showed reversible inhibition of CYP3A4, not CYP2J2. Three TKIs also showed time-dependent inactivation of CYP3A4. Notably, sunitinib had the strongest inactivation effect on CYP3A4 than the other TKIs with a 4.00-fold $\mathrm{IC}_{50}$ shift, however, a slight effect on CYP2J2. Docking simulations revealed the relation of inhibitory activity to ChemScore. Additionally, drug-drug interaction risks of combinations were assessed using pharmacokinetic data of cancer patients. Imatinib, which showed the strongest inhibition, was predicted to cause a 114-244\% increase in rivaroxaban exposure.

Conclusion and Implications: Imatinib was predicted to have a moderate DDI risk when was combined with rivaroxaban. These results provide evidence for medication guidance when combining rivaroxaban with TKIs for cancer patients, and also give new insight for the DDI assessment involving rivaroxaban.

Keywords:combined medication, CYP2J2, CYP3A4, drug-drug interaction, medication safety

\title{
Introduction
}

Cancer patients are one of the target populations of anticoagulation. Cancer-related vein thromboembolism (VTE) is closely associated with increased morbidity and mortality, and thrombotic diseases have been the leading cause of non-neoplastic death in cancer patients (Song, Rosovsky, Connors \& Al-Samkari, 2019; Timp, Braekkan, Versteeg \& Cannegieter, 2013; Zamorano et al., 2016). According to statistics, cancer patients have an approximately 4-7-fold higher risk of VTE than normal patients, and cancer patients with VTE account for about 20\% of all VTE patients (Blom, Doggen, Osanto \& Rosendaal, 2005; Streiff, 2016). Compared with non-cancer patients, the recurrence rate of VTE in cancer patients is 3-4 times higher and the incidence of major bleeding is also increased by 2-3 times (Douketis, Crowther, Foster \& Ginsberg, 2001; Levitan et al., 1999; Monreal et al., 2006; Prandoni et al., 2002). In cancer patients, the presence of tumours is one of the major risk factors for VTE. Also, both the hypercoagulability of cancer patients and the thrombogenicity of anti-cancer agents are reasons (Shalhoub et al., 2017; Zamorano et al., 2016). In addition to VTE, the risk of atrial fibrillation (AF) for cancer patients was also extremely high (Onaitis, D'Amico, Zhao, O'Brien \& Harpole, 2010). An analysis showed that the occurrence of AF for cancer patients in the 90 days after the cancer diagnosis was much higher than for normal patients (Saliba, Rennert, Gronich, Gruber \& Rennert, 2018). And notably, the prevention of stroke and systemic embolism by using anticoagulants is one of the therapeutic cornerstones of AF management (January et al., 2019). Therefore, coagulation 
prevention or anticoagulant treatment is unavoidable in cancer patients. Thus, cancer patients may receive anticoagulant therapy concurrently with anti-cancer therapy.

For decades, anticoagulant therapy for cancer-related VTE has been limited to vitamin K antagonists (VKAs) and heparin drugs. Recently, direct oral anticoagulants (DOACs) have also become an option (Streiff et al., 2020). Rivaroxaban is recommended for the treatment of superficial vein thrombosis and VTE prophylaxis following the discharge by the National Comprehensive Cancer Network of America in 2020 of the clinical practice guidelines for cancer-associated venous thromboembolic disease (Streiff et al., 2020). Additionally, data from large randomised clinical trials also demonstrated that DOCAs combined with validated risk assessment scores are a reasonable choice for the primary thromboprophylaxis of cancer patients instead of low molecular weight heparin (Song, Rosovsky, Connors \& Al-Samkari, 2019). Furthermore, the ease of taking this medicine improves patient adherence. Thus, DOCAs, especially rivaroxaban, may become an alternative to the standard therapy for cancer patients.

The co-medication safety assessment of rivaroxaban is still lacking. Rivaroxaban is widely used for the prevention and treatment of thromboembolic disorders in clinical practice. Although in vivo pharmacokinetic data are stable, bleeding is still a risk factor that should be considered for medication safety. Our previous studies have shown CYP2J2 and CYP3A4 to be the major isoforms involved in the metabolic process of rivaroxaban, and importantly CYP2J2 showed ${ }^{39}$-fold higher catalytic efficiency than CYP3A4 (Zhao et al., 2021). CYP3A4 is always considered as the enzyme which resulted in drug-drug interactions (DDIs), however, CYP2J2 is always ignored due to its lower abundance in the liver. CYP2J2 is a P450 isoform that is mainly distributed in the heart and arteries, which is responsible for the metabolism of arachidonic acid. Notably, CYP2J2 was recently highlighted as an emerging tumour marker. Numerous studies have reported the high expression of CYP2J2 in various cancer cell lines, tumour tissues and even in the liver of cancer patients, which may relate to tumour expansion and metastasis (Allison et al., 2017; Karkhanis, Hong \& Chan, 2017). Therefore, the status of CYP2J2 in rivaroxaban DDI research cannot be ignored. However, safety assessment data on medication combinations are lacking, so the related roles of CYP2J2 are unknown.

Tyrosine kinase inhibitors (TKIs) are the most commonly used drugs against cancers clinically. Among these, imatinib, sunitinib and gefitinib have been the mainstay treatments for various solid tumours since their launch in 2000 (Burotto, Manasanch, Wilkerson \& Fojo, 2015; Cheng et al., 2013; Kuczynski, Lee, Man, Chen \& Kerbel, 2015; Tirumani, Jagannathan, Krajewski, Shinagare, Jacene \& Ramaiya, 2013; Wertheimer et al., 2015). Imatinib was almost the first TKI anti-tumour drug to gain approval by the US Food and Drug Administration (FDA), and imatinib has become a first-line clinical drug for gastrointestinal stromal tumours (GIST) (O'Brien et al., 2003; von Mehren \& Widmer, 2011). However, due to the long treatment cycles, the safety of imatinib in combination with other drugs is particularly important (Guilhot, 2004; Nebot, Crettol, d'Esposito, Tattam, Hibbs \& Murray, 2010). As a multitargeted TKI, sunitinib exerts strong angiogenesis inhibitory activity. It was approved by the FDA in 2006 as a first-line drug for metastatic renal cell carcinoma, and it was also used as a second-line drug for imatinib-resistant patients (Kalra, Rini \& Jonasch, 2015). Gefitinib was the first TKI to gain approval in the US and Japan for treating advanced non-small-cell lung cancer (NSCLC), and it can significantly prolong the progression-free survival of NSCLC patients (Dhillon, 2015). It is noteworthy that CYP3A4 accounts for a considerable proportion of the CYPmediated metabolism of these three TKIs, which is similar to rivaroxaban. Indeed, it is the overlap between the metabolic enzymes for rivaroxaban and these three TKIs that may produce DDIs.

The guidelines of combinations of DOCAs with other drugs were always cautious. American Society of Hematology recommended that DOCAs should be replaced with other anticoagulation when in coadministration with strong CYP inducers or inhibitors based on low certainty in effectiveness and safety (Witt et al., 2018). But it is unreasonable to completely deny DOCAs combinations in clinical practice. Imatinib, sunitinib and gefitinib have been widely applied for patients with solid tumours. Thus, the comitant administration of rivaroxaban with these three TKIs has a profound combination foundation in the treatment of cancer patients, and the safety of this combination deserves further attention. However, assessment data on the safety of rivaroxaban with TKIs is limited. Hence, more relevant and detailed pharmacokinetics measurements are 
required. The present study assessed the DDI risk of the combination of rivaroxaban with the three TKIs by in vitro enzyme assays. Importantly, the investigation was mainly performed on CYP2J2 and CYP3A4 to comprehensively explore their reversible and time-dependent inactivation behaviours. Finally, the in vivo DDI risk of the combination of rivaroxaban with the three TKIs was estimated according to detailed pharmacokinetic parameters of cancer patients, producing direct evidence to inform clinical medication safety assessment.

\section{Methods}

\section{Materials and chemicals}

Rivaroxaban, sunitinib and NADPH were purchased from Shanghai Yuanye Bio-Technology Co., Ltd. Imatinib and gefitinib were obtained from Sigma-Aldrich (Missouri, USA). cDNA-expressed recombinant human CYP3A4 was purchased from Cypex Ltd (Dundee, UK). Pooled human liver microsomes (HLM) and recombinant human CYP2J2 were purchased from BioreclamationIVT (Maryland, USA). All analytical reagent grade and HPLC grade solvents were from Tedia, Inc. (Ohio, USA). High-performance liquid chromatography (HPLC) was performed using an Agilent MSD/MS system controller, two 1260 series pumps, a 1200 series autosampler and a 1200 series variable wavelength detector.

\section{Analytical methods}

The inhibitory effect of the TKIs on the metabolism of rivaroxaban in recombined P450 isoforms and pooled human liver microsomes were compared by quantifiably detecting the production of the major metabolite using HPLC. The detection and quantitative analysis were achieved by HPLC, as described in our previous study (Zhao et al., 2021).

\section{Enzyme inhibition assays}

The concentration of HLM, CYP2J2 and CYP3A4 was $0.3,0.4$ and $0.6 \mathrm{mg} \cdot \mathrm{mL}^{-1}$. The selection of the rivaroxaban concentration depended on the $\mathrm{K}_{\mathrm{m}}$ values of the kinetic studies $(22.81,19.37$ and $46.98 \mu \mathrm{M}$ for HLM, CYP2J2 and CYP3A4, respectively) (Zhao et al., 2021). The concentration of CYP3A4 (0.6 mg. $\mathrm{mL}^{-1}$ ) was selected to correspond with the lowest detected concentration of M1. The detailed method can be found in our previous publication (Zhao et al., 2021).

\section{Reversible inhibition of CYP3A4 and CYP2J2 by TIKs}

The concentration range of rivaroxaban in incubation of CYP2J2 and CYP3A4 was 0-100 $\mu \mathrm{M}$ and 0-400 $\mu \mathrm{M}$ respectively. The inhibition constant $\left(K_{i}\right)$ was determined using various concentrations of inhibitors and rivaroxaban. $\mathrm{K}_{i}$ was calculated by three inhibition mode formulae (competitive, non-competitive and mixedmode) using Prism v.6.0 (GraphPad, San Diego, CA, USA). Detailed information on the fitting formulae and related parameters can be found in our previous publication ( $\mathrm{Li}, \mathrm{Cao}, \mathrm{He}, \mathrm{Ge}, \mathrm{Guo} \& \mathrm{Wu}, 2018$ ).

$I C_{50}$ shift assay

The 30 min pre-incubation of TKIs with NADPH and CYP2J2 or CYP3A4 preceded the normal incubation, following which the $\mathrm{IC}_{50}$ values $\left(\mathrm{IC}_{50}\right.$ shift) were re-determined. These $\mathrm{IC}_{50}$ shift values were compared with the $\mathrm{IC}_{50}$ values that were determined without the 30 min pre-incubation, with a more than 1.5 -fold decrease considered to be evidence of time-dependent inactivation. Other reaction conditions were as mentioned above.

\section{Time-dependent inactivation of CYP3A4}

To investigate the time-dependent inactivation of CYP3A4 by sunitinib, seven gradient concentrations $(0-5$ $\mu \mathrm{M})$ and six time points $(0-20 \mathrm{~min})$ were used. The data were then fitted to a linear regression model, which reflected the linear relation between 'ln remaining activity' and 'inactivation concentration' $(I)$. The negative slope of this linear relationship reflected the observed inactivation rates $\left(\mathrm{K}_{\mathrm{obs}}\right)$ value, which could be plotted against $I$ to allow the fitting of inactivation kinetic parameters $K_{\mathrm{I}}$ and $K_{\text {inact }}$ to the nonlinear least-squares regression based on Eq. 1. using Prism v.6.0 (GraphPad, San Diego, CA, USA). 


\section{(1)}

\section{Molecular docking simulations}

The CYP2J2 crystal structure homology model from the Clustal Omega webserver (https://www.ebi.ac.uk/Tools/msa/clustalo/) was used to conduct docking simulations between TKIs and rivaroxaban in SYBYL (X-1.1) (Ning et al., 2019). The crystal structure of CYP3A4 (PDB: 4D7D) was from the crystal structures that bound to a known inhibitor. The 3D structures of the TKIs were subjected to energy minimisation using the default Tripos force field parameters, and the Gasteiger-Hückel charges were calculated for each compound. The Surflex-Dock mode was used to generate binding conformations of TKIs with CYP2J2. The optimal conformations were determined by their empirical functions ChemScore. The PyMOL Molecular Graphics System v.16.1.0.15350 (DeLano Scientific LLC) was used to visualise the docking results.

\section{Quantitative prediction of DDI risk}

Kinetic constants were included in the mechanistic static model to explore reversible inhibition and timedependent inactivation. This static model was previously developed and refined by Fahmi et al. (Fahmi, Maurer, Kish, Cardenas, Boldt \& Nettleton, 2008) and Isoherranen et al. (Isoherranen, Lutz, Chung, Hachad, Levy \& Ragueneau-Majlessi, 2012) to account for the inhibition of multiple P450 isoforms. In the present study, this model was designed to explore the contributions of enzyme inhibition in the prediction of DDI risk. The area under the curve ratio $(\mathrm{AUC}$ Ratio/AUC $\mathrm{R}$ ) in the presence of a pharmacokinetic DDI was used as the index, as described by Eq. 2 .

\section{(2)}

Here, A is the time-dependent inactivation of each P450 isoform that was observed in the liver, as described by Eq. 3.

\section{(3) (4)}

Here, B is the reversible inhibition of each $\mathrm{P} 450$ isoform that was observed in the liver, as described by Eq. 4. The degradation rates $\left(\mathrm{K}_{\mathrm{deg}}\right)$ of CYP2J2 and CYP3A4 were 0.00026 and $0.00032 \mathrm{~min}^{-1}$, respectively (Cheong et al., 2017), where I represented the in vivo concentration of inhibitors in healthy and solid tumour patients. Additionally, the fraction of rivaroxaban metabolised by CYP2J2 or CYP3A4 was input from our previous study (Zhao et al., 2021), which were 0.95 for CYP2J2 and 0.025 for CYP3A4.

\section{Results}

\section{Inhibition of TKIs on rivaroxaban metabolism}

Three TKI concentrations $(1,10$, and $100 \mu \mathrm{M})$ were used to investigate the inhibitory intensity. Imatinib and gefitinib showed strong inhibition of the metabolism of rivaroxaban, but sunitinib only exerted inhibitory activity in the incubation with HLM and CYP3A4. Imatinib exerted the strongest inhibitory effect on CYP3A4-mediated rivaroxaban metabolism, with undetectable formation of M1 in the incubation with 100 $\mu \mathrm{M}$ imatinib (Figure 1B). Gradient concentrations were used to determine the $\mathrm{IC}_{50}$ values. In CYP3A4mediated rivaroxaban metabolism, imatinib showed the strongest inhibitory activity with an $\mathrm{IC}_{50}$ value of $4.35 \mu \mathrm{M}$, and gefitinib showed the strongest inhibitory effect on metabolism by CYP2J2 with an $\mathrm{IC}_{50}$ value of $3.72 \mu \mathrm{M}$. In the metabolism associated with HLM incubation, imatinib was also the strongest inhibitor with an $\mathrm{IC}_{50}$ value of $1.70 \mu \mathrm{M}$. Broadly, both imatinib and gefitinib showed a strong inhibitory effect on the rivaroxaban metabolism mediated by CYP3A4 and CYP2J2. In contrast, sunitinib only exerted an inhibitory effect against CYP3A4-mediated metabolism, while the effect on CYP2J2-mediated metabolism was not obvious with an $\mathrm{IC}_{50}$ value of $397.70 \mu \mathrm{M}$ (Figure $2 \mathrm{E}, \mathrm{F}$ ). The detailed $\mathrm{IC}_{50}$ values are shown in Table 1.

Reversible inhibition behaviour of TKIs on CYP3A4 and CYP2J2 
The $\mathrm{K}_{i}$ value of the TKIs was fitted from the kinetic curve and the $\mathrm{R}^{2}$ values and inhibition modes are shown in Tables 2. As sunitinib did not exert more than $50 \%$ inhibition even at $250 \mu \mathrm{M}$, the $\mathrm{K}_{i}$ value was not measured. All three TKIs showed non-competitive inhibition on CYP3A4- and CYP2J2-mediated rivaroxaban metabolism with good correlation (Figure 3 and 4). This was corroborated by the respective Dixon and Lineweaver-Burk plots. As with the $\mathrm{IC}_{50}$ results, imatinib showed the strongest inhibition on CYP3A4-mediated rivaroxaban metabolism with a $\mathrm{K}_{i}$ value of $1.92 \mu \mathrm{M}$. The $\mathrm{K}_{i}$ values of sunitinib and gefitinib with CYP3A4 were 13.24 and $4.91 \mu \mathrm{M}$, respectively, which were similar to their $\mathrm{IC}_{50}$ values.

$I_{50}$-shift assays on CYP2J2 and CYP3A4

$\mathrm{IC}_{50}$ shift assays of CYP2J2 and CYP3A4 were performed to explore the mechanism-dependent inhibition. Compared with direct inhibition, the 30-min pre-incubation with NADPH in the CYP2J2 incubation did not significantly change the inhibitory effect of imatinib and gefitinib (Table 3). While the inhibitory effect of sunitinib was slightly increased, which resulted in 4.00 -fold decrease in the $\mathrm{IC}_{50}$ value (Figure $5 \mathrm{~B}$ and Table 3). In brief, the 30-min pre-incubation did not significantly affect the inhibition of CYP2J2 by the three TKIs, with all $\mathrm{IC}_{50}$ shifts being less than 1.5 -fold. In contrast, all $\mathrm{IC}_{50}$ values for the inhibition of CYP3A4 by the TKIs decreased by more than 1.5-fold (Table 3). Notably, sunitinib showed the largest change in $\mathrm{IC}_{50}$ shift (Figure $5 \mathrm{E}$ ), with an $\mathrm{IC}_{50}$ value decreasing to $2.73 \mu \mathrm{M}$ following the 30 min pre-incubation.

Time-dependent inactivation of CYP3A4 by sunitinib

Given the 4.00-fold $\mathrm{IC}_{50}$ shift of sunitinib on CYP3A4 following 30-min pre-incubation with NADPH, timedependent inactivation constants of sunitinib were further determined (Figure 6A). The maximum inactivation rate $\left(\mathrm{K}_{\text {inact }}\right)$ and the inhibitor concentration needed to cause half of $\mathrm{K}_{\text {inact }}\left(\mathrm{K}_{\mathrm{I}}\right)$ were fitted using the non-linear regression method. As shown in Figure $6 \mathrm{~B}$, the $\mathrm{K}_{\text {inact }}$ and $\mathrm{K}_{\mathrm{I}}$ values of sunitinib were $0.0339 \mathrm{~min}^{-1}$ and $2.901 \mu \mathrm{M}$, respectively. The $\mathrm{K}_{\text {inact }}$ value indicated that approximately $3.4 \%$ of CYP3A4 was inactivated per minute when it was incubated with the saturating concentration of sunitinib.

\section{Molecular docking simulations}

Molecular docking simulations were used to elucidate the binding conformations for the interactions between the TKIs and CYP2J2 or CYP3A4. In the docking simulation between CYP2J2 and the TKIs, gefitinib had the lowest ChemScore value, followed by imatinib and then sunitinib. This ChemScore ranking was consistent with the inhibitory intensity of the TKIs on CYP2J2. Additionally, imatinib had the lowest ChemScore value in the docking simulation with CYP3A4, followed by gefitinib and then sunitinib, which was also consistent with the inhibitory intensity of these TKIs on CYP3A4 (Figure 7).

Estimation of the DDI risk between rivaroxaban and the three TKIs

According to the inhibition constants of the TKIs for CYP2J2- and CYP3A4-mediated rivaroxaban metabolism, the AUC fold-changes when the TKIs were combined with rivaroxaban were predicted. Imatinib was predicted to at most result in $244 \%$ increase in rivaroxaban exposure (Table 4). Sunitinib and gefitinib were predicted to at most result in rivaroxaban exposure increases of $2 \%$ and $11 \%$, respectively.

\section{Discussion}

All three TKIs were found to have a remarkable inhibitory effect on CYP3A4-mediated rivaroxaban metabolism. Imatinib showed the strongest reversible inhibitory effect towards CYP3A4 with a $\mathrm{K}_{i}$ value of $1.92 \mu \mathrm{M}$. The inhibition of CYP3A4 by sunitinib or gefitinib was also potent, with $\mathrm{K}_{i}$ values of 13.24 and $4.91 \mu \mathrm{M}$, respectively. In addition to reversible inhibition, the three TKIs also exerted time-dependent inactivation of CYP3A4, especially sunitinib. Compared with reversible inhibition, mechanism-dependent inactivation is always more frequently related to unfavourable DDIs in clinical practice (Kalgutkar, Obach \& Maurer, 2007). Importantly, the expression of CYP3A4 which is abundantly expressed in the liver showed more than 100-fold population variability (Zanger \& Schwab, 2013). More importantly, it has been reported that sunitinib has a 10-fold higher concentration in the liver than in blood (Lau et al., 2015). Thus, being cautious about the CYP3A4 enzyme expression in patients who take both rivaroxaban and TKIs is necessary. 
The present study found that CYP2J2, which dominates the metabolism of rivaroxaban, was inhibited by the TKIs. Imatinib and gefitinib were potent inhibitors of CYP2J2 with $\mathrm{K}_{i}$ values of 3.53 and $2.99 \mu \mathrm{M}$, respectively. Additionally, there was no irreversible inhibition of CYP2J2 by the three TKIs, with the results showing $\mathrm{IC}_{50}$ shifts of less than 1.5-fold. Although the three TKIs did not irreversibly inactivate CYP2J2, the DDI risk produced by inhibiting CYP2J2 cannot be ignored due to the distribution of CYP2J2 in vivo. CYP2J2 was identified as an enzyme that mainly distributed in the heart (Das, Weigle, Arnold, Kim, Carnevale \& Huff, 2020): the mRNA levels of CYP2J2 in the cardiovascular system exceed those of other detected isozymes by 3 million to 62 times (Michaud, Frappier, Dumas \& Turgeon, 2010; Wu, Moomaw, Tomer, Falck \& Zeldin, 1996). Although CYP2J2 is not usually considered as a DDI target due to its lower content in the liver, if the heart is set as the organ for potential DDIs, there may be an extremely high risk of DDI between rivaroxaban and TKIs. In addition to its physiological distribution in the cardiovascular system, CYP2J2 has also recently been found to be highly expressed in various tumour tissues (Allison et al., 2017; Karkhanis, Hong \& Chan, 2017). Moreover, TKIs would accumulate in the tumour tissue, thus the DDI risks of rivaroxaban in combination with TKIs for cancer patients may be higher than in our predictions. Therefore, it is necessary to include CYP2J2 in DDI research of rivaroxaban.

Imatinib was predicted to have moderate DDI risk when combined with rivaroxaban. Based on the inhibitory constants of imatinib on CYP2J2 and CYP3A4, and the metabolic contributions of two isoforms, imatinib was predicted to yield at most a 2.44-fold rivaroxaban AUC increase. Therefore, according to the FDA's guidelines for the relative risk of DDIs, a moderate DDI risk may exist for the combination of rivaroxaban and imatinib (Table 4). This result was predicted from the pharmacokinetic data of patients with solid tumours who were received TKIs. Notably, cancer patients have poorer metabolic function than normal patients, which may directly lead to higher plasma concentrations of TKIs. More importantly, patients who are refractory to standard therapy have the much higher plasma concentrations (Scheffler, Di Gion, Doroshyenko, Wolf \& Fuhr, 2011). Therefore, using pharmacokinetic data from cancer patients may make our prediction more accurate. However, metabolic enzyme activity is subject to individual variability, so factors such as hepatic blood flow and genetic polymorphism cannot be neglected (Zanger \& Schwab, 2013). Factors that influence the enzyme activity of cancer patients may be more difficult to determine due to the more complicated in vivo environment. All these factors would produce great diversity in the pharmacokinetic data of TKIs in cancer patients. Therefore, an individual physiologically-based pharmacokinetic model for cancer patients would be encouraged in the prediction of the pharmacokinetic behaviour of rivaroxaban in combination with TKIs.

\section{Conclusion}

In summary, all three TKIs (imatinib, sunitinib and gefitinib) showed inhibitory effects on CYP2J2- and CYP3A4-mediated rivaroxaban metabolism. Imatinib and gefitinib exerted significant reversible inhibition of CYP2J2 and CYP3A4, while sunitinib only showed reversible inhibition of CYP3A4. The three TKIs also demonstrated time-dependent inactivation of CYP3A4, with this effect being slight on CYP2J2. Furthermore, the combination of rivaroxaban with imatinib was predicted to constitute a moderate DDI risk. Our results provide data for the clinical safety assessment of the combination of rivaroxaban with imatinib, sunitinib and gefitinib in cancer patients, and also give new insights for DDI assessment involving rivaroxaban.

\section{Data Availability}

The data underlying this article will be shared on reasonable request to the corresponding author.

\section{Author Contributions}

Tingting Zhao : Software, Validation, Formal analysis, Investigation, Writing-Original Draft. Xuening Li : Software, Validation, Formal analysis, Investigation, Writing Original Draft.Yanwei Chen: Software, Validation, Formal analysis, Investigation, Writing Original Draft. Jingjing Wu : Conceptualization, Supervision, Writing-review and editing.Dalong Wang: Supervision, Data Curation. Shan Zhao : Supervision, Data Curation. Liyan Wang : Supervision.Changyuan Wang : Project administration. Qiang 
Meng : Supervision. Huijun Sun : Supervision. Kexin Liu : Supervision.

\section{Funding}

This work was supported by the National Natural Science Foundation of China (81874324, 81922070, 81973286, 81803627, 81702995 and U1608283), and LiaoNing Revitalization Talents Program (XLYC1907103).

Conflict of interest: none declared.

\section{References}

Allison SE, Chen Y, Petrovic N, Zhang J, Bourget K, Mackenzie PI, et al. (2017). Activation of ALDH1A1 in MDA-MB-468 breast cancer cells that over-express CYP2J2 protects against paclitaxel-dependent cell death mediated by reactive oxygen species. Biochem Pharmacol 143: 79-89.

Blom JW, Doggen CJ, Osanto S, \& Rosendaal FR (2005). Malignancies, prothrombotic mutations, and the risk of venous thrombosis. Jama 293: 715-722.

Burotto M, Manasanch EE, Wilkerson J, \& Fojo T (2015). Gefitinib and erlotinib in metastatic non-small cell lung cancer: a meta-analysis of toxicity and efficacy of randomized clinical trials. Oncologist 20: 400-410.

Cheng AL, Kang YK, Lin DY, Park JW, Kudo M, Qin S, et al. (2013). Sunitinib versus sorafenib in advanced hepatocellular cancer: results of a randomized phase III trial. Journal of clinical oncology : official journal of the American Society of Clinical Oncology 31:4067-4075.

Cheong EJ, Goh JJ, Hong Y, Venkatesan G, Liu Y, Chiu GN, et al.(2017). Application of Static Modeling in the Prediction of In Vivo Drug-Drug Interactions between Rivaroxaban and Antiarrhythmic Agents Based on In Vitro Inhibition Studies. Drug Metab Dispos 45:260-268.

Das A, Weigle AT, Arnold WR, Kim JS, Carnevale LN, \& Huff HC (2020). CYP2J2 Molecular Recognition: A New Axis for Therapeutic Design. Pharmacol Ther 215: 107601.

Dhillon S (2015). Gefitinib: a review of its use in adults with advanced non-small cell lung cancer. Target Oncol 10: 153-170.

Douketis JD, Crowther MA, Foster GA, \& Ginsberg JS (2001). Does the location of thrombosis determine the risk of disease recurrence in patients with proximal deep vein thrombosis? Am J Med 110:515-519.

Fahmi OA, Maurer TS, Kish M, Cardenas E, Boldt S, \& Nettleton D (2008). A combined model for predicting CYP3A4 clinical net drug-drug interaction based on CYP3A4 inhibition, inactivation, and induction determined in vitro. Drug Metab Dispos 36: 1698-1708.

Guilhot F (2004). Indications for imatinib mesylate therapy and clinical management. Oncologist 9: 271-281.

Isoherranen N, Lutz JD, Chung SP, Hachad H, Levy RH, \& Ragueneau-Majlessi I (2012). Importance of multi-p450 inhibition in drug-drug interactions: evaluation of incidence, inhibition magnitude, and prediction from in vitro data. Chem Res Toxicol 25:2285-2300.

January CT, Wann LS, Calkins H, Chen LY, Cigarroa JE, Cleveland JC, Jr., et al. (2019). 2019 AHA/ACC/HRS Focused Update of the 2014 AHA/ACC/HRS Guideline for the Management of Patients With Atrial Fibrillation: A Report of the American College of Cardiology/American Heart Association Task Force on Clinical Practice Guidelines and the Heart Rhythm Society in Collaboration With the Society of Thoracic Surgeons. Circulation 140: e125-e151.

Kalgutkar AS, Obach RS, \& Maurer TS (2007). Mechanism-based inactivation of cytochrome P450 enzymes: chemical mechanisms, structure-activity relationships and relationship to clinical drug-drug interactions and idiosyncratic adverse drug reactions. Curr Drug Metab 8: 407-447. 
Kalra S, Rini BI, \& Jonasch E (2015). Alternate sunitinib schedules in patients with metastatic renal cell carcinoma. Ann Oncol 26:1300-1304.

Karkhanis A, Hong Y, \& Chan ECY (2017). Inhibition and inactivation of human CYP2J2: Implications in cardiac pathophysiology and opportunities in cancer therapy. Biochem Pharmacol 135: 12-21.

Kuczynski EA, Lee CR, Man S, Chen E, \& Kerbel RS (2015). Effects of Sorafenib Dose on Acquired Reversible Resistance and Toxicity in Hepatocellular Carcinoma. Cancer Res 75: 2510-2519.

Lau CL, Chan ST, Selvaratanam M, Khoo HW, Lim AY, Modamio P, et al. (2015). Sunitinib-ibuprofen drug interaction affects the pharmacokinetics and tissue distribution of sunitinib to brain, liver, and kidney in male and female mice differently. Fundamental \& clinical pharmacology 29: 404-416.

Levitan N, Dowlati A, Remick SC, Tahsildar HI, Sivinski LD, Beyth R, et al. (1999). Rates of initial and recurrent thromboembolic disease among patients with malignancy versus those without malignancy. Risk analysis using Medicare claims data. Medicine (Baltimore) 78: 285-291.

Li JN, Cao YF, He RR, Ge GB, Guo B, \& Wu JJ (2018). Evidence for Shikonin acting as an active inhibitor of human carboxylesterases 2: Implications for herb-drug combination. Phytother Res 32:1311-1319.

Michaud V, Frappier M, Dumas MC, \& Turgeon J (2010). Metabolic activity and mRNA levels of human cardiac CYP450s involved in drug metabolism. PLoS One 5: e15666.

Monreal M, Falgá C, Valdés M, Suárez C, Gabriel F, Tolosa C, et al. (2006). Fatal pulmonary embolism and fatal bleeding in cancer patients with venous thromboembolism: findings from the RIETE registry. J Thromb Haemost 4: 1950-1956.

Nebot N, Crettol S, d'Esposito F, Tattam B, Hibbs DE, \& Murray M (2010). Participation of CYP2C8 and CYP3A4 in the N-demethylation of imatinib in human hepatic microsomes. Br J Pharmacol 161:1059-1069.

Ning J, Liu T, Dong P, Wang W, Ge G, Wang B, et al. (2019). Molecular Design Strategy to Construct the Near-Infrared Fluorescent Probe for Selectively Sensing Human Cytochrome P450 2J2. J Am Chem Soc 141: 1126-1134.

O'Brien SG, Guilhot F, Larson RA, Gathmann I, Baccarani M, Cervantes F, et al. (2003). Imatinib compared with interferon and low-dose cytarabine for newly diagnosed chronic-phase chronic myeloid leukemia. N Engl J Med 348: 994-1004.

Onaitis M, D'Amico T, Zhao Y, O'Brien S, \& Harpole D (2010). Risk factors for atrial fibrillation after lung cancer surgery: analysis of the Society of Thoracic Surgeons general thoracic surgery database. Ann Thorac Surg 90: 368-374.

Prandoni P, Lensing AW, Piccioli A, Bernardi E, Simioni P, Girolami B, et al. (2002). Recurrent venous thromboembolism and bleeding complications during anticoagulant treatment in patients with cancer and venous thrombosis. Blood 100: 3484-3488.

Saliba W, Rennert HS, Gronich N, Gruber SB, \& Rennert G (2018). Association of atrial fibrillation and cancer: Analysis from two large population-based case-control studies. PLoS One 13: e0190324.

Scheffler M, Di Gion P, Doroshyenko O, Wolf J, \& Fuhr U (2011). Clinical pharmacokinetics of tyrosine kinase inhibitors: focus on 4-anilinoquinazolines. Clin Pharmacokinet 50: 371-403.

Shalhoub J, Norrie J, Baker C, Bradbury AW, Dhillon K, Everington T, et al. (2017). Graduated Compression Stockings as an Adjunct to Low Dose Low Molecular Weight Heparin in Venous Thromboembolism Prevention in Surgery: A Multicentre Randomised Controlled Trial. Eur J Vasc Endovasc Surg 53: 880-885.

Song AB, Rosovsky RP, Connors JM, \& Al-Samkari H (2019). Direct oral anticoagulants for treatment and prevention of venous thromboembolism in cancer patients. Vascular health and risk management 15:175-186. 
Streiff MB (2016). Thrombosis in the setting of cancer. Hematology Am Soc Hematol Educ Program 2016: 196-205.

Streiff MB, Holmstrom B, Angelini D, Ashrani A, Bockenstedt PL, Chesney C, et al. (2020). NCCN Guidelines Insights: Cancer-Associated Venous Thromboembolic Disease, Version 1.2020. J Natl Compr Canc Netw 16: 1289-1303.

Timp JF, Braekkan SK, Versteeg HH, \& Cannegieter SC (2013). Epidemiology of cancer-associated venous thrombosis. Blood 122:1712-1723.

Tirumani SH, Jagannathan JP, Krajewski KM, Shinagare AB, Jacene H, \& Ramaiya NH (2013). Imatinib and beyond in gastrointestinal stromal tumors: A radiologist's perspective. AJR Am J Roentgenol 201:801810.

von Mehren M, \& Widmer N (2011). Correlations between imatinib pharmacokinetics, pharmacodynamics, adherence, and clinical response in advanced metastatic gastrointestinal stromal tumor (GIST): an emerging role for drug blood level testing? Cancer Treat Rev 37:291-299.

Wertheimer C, Siedlecki J, Kook D, Mayer WJ, Wolf A, Klingenstein A, et al. (2015). EGFR inhibitor Gefitinib attenuates posterior capsule opacification in vitro and in the ex vivo human capsular bag model. Graefes Arch Clin Exp Ophthalmol 253: 409-417.

Witt DM, Nieuwlaat R, Clark NP, Ansell J, Holbrook A, Skov J, et al. (2018). American Society of Hematology 2018 guidelines for management of venous thromboembolism: optimal management of anticoagulation therapy. Blood advances 2: 3257-3291.

Wu S, Moomaw CR, Tomer KB, Falck JR, \& Zeldin DC (1996). Molecular cloning and expression of CYP2J2, a human cytochrome P450 arachidonic acid epoxygenase highly expressed in heart. J Biol Chem 271:34603468 .

Zamorano JL, Lancellotti P, Rodriguez Muñoz D, Aboyans V, Asteggiano R, Galderisi M, et al. (2016). 2016 ESC Position Paper on cancer treatments and cardiovascular toxicity developed under the auspices of the ESC Committee for Practice Guidelines: The Task Force for cancer treatments and cardiovascular toxicity of the European Society of Cardiology (ESC). Eur Heart J 37: 2768-2801.

Zanger UM, \& Schwab M (2013). Cytochrome P450 enzymes in drug metabolism: regulation of gene expression, enzyme activities, and impact of genetic variation. Pharmacol Ther 138: 103-141.

Zhao T, Chen Y, Wang D, Wang L, Dong P, Zhao S, et al. (2021). Identifying the Dominant Contribution of Human Cytochrome P450 2J2 to the Metabolism of Rivaroxaban, an Oral Anticoagulant. Cardiovasc Drugs Ther.

\section{Figure Legends}

Figure 1. The initial screening of imatinib, sunitinib and gefitinib inhibition with: (A) HLM, (B) CYP2J2 and (C) CYP3A4. The TKI concentrations were 1, 10 and $100 \mu \mathrm{M}$. Results are shown as the mean \pm S.D. of at least five determinations. N.D.: not detectable.

Figure 2. Dose-response curves of TKI inhibition with (A, D) HLM, (B, E) CYP2J2 and (C, F) CYP3A4. Results are shown as the mean from at least five experiments.

Figure 3. Reversible inhibition of CYP2J2 by imatinib and gefitinib. Lineweaver-Burk plots for the inhibition of (A) imatinib and (C) gefitinib (C) on CYP2J2-mediated rivaroxaban metabolism; (B) and (D) are the corresponding Dixon plots. Data represent the mean \pm S.D.

Figure 4. Reversible inhibition of CYP3A4 by imatinib, gefitinib and sunitinib. Lineweaver-Burk plots for the inhibition of (A) imatinib, (C) gefitinib and (E) sunitinib on CYP3A4-mediated rivaroxaban metabolism; (B), (D) and (F) are the corresponding Dixon plots. Data represent the mean \pm S.D. 
Figure 5. Effects of imatinib, sunitinib and gefitinib on rivaroxaban metabolism by (A, B and C) CYP2J2 and (D, E and F) CYP3A4 with or without a 30-min pre-incubation in the presence of NADPH. Data points are from five independent experiments.

Figure 6. (A) Time- and concentration-dependent inactivation by sunitinib of CYP3A4-mediated rivaroxaban metabolism. (B) Observed inactivation rates $\left(\mathrm{K}_{\text {obs }}\right)$ were plotted against the sunitinib concentration to calculate the inactivation kinetic constants $\mathrm{K}_{I}$ and $\mathrm{K}_{\text {inact }}$.

Figure 7. Molecular docking simulations of imatinib (A), sunitinib (B) and gefitinib (C) with CYP2J2, and (D, E and F) with CYP3A4.

\section{Hosted file}

Table1.docx available at https://authorea.com/users/363498/articles/528479-a-combinedmedication-safety-assessment-of-rivaroxaban-with-tyrosine-kinase-inhibitors-for-cancerpatients-focusing-on-cyp2j2-and-cyp3a4

\section{Hosted file}

Table2.docx available at https://authorea.com/users/363498/articles/528479-a-combinedmedication-safety-assessment-of-rivaroxaban-with-tyrosine-kinase-inhibitors-for-cancerpatients-focusing-on-cyp2j2-and-cyp3a4

\section{Hosted file}

Table3.docx available at https://authorea.com/users/363498/articles/528479-a-combinedmedication-safety-assessment-of-rivaroxaban-with-tyrosine-kinase-inhibitors-for-cancerpatients-focusing-on-cyp2j2-and-cyp3a4

\section{Hosted file}

Table4.docx available at https://authorea.com/users/363498/articles/528479-a-combinedmedication-safety-assessment-of-rivaroxaban-with-tyrosine-kinase-inhibitors-for-cancerpatients-focusing-on-cyp2j2-and-cyp3a4
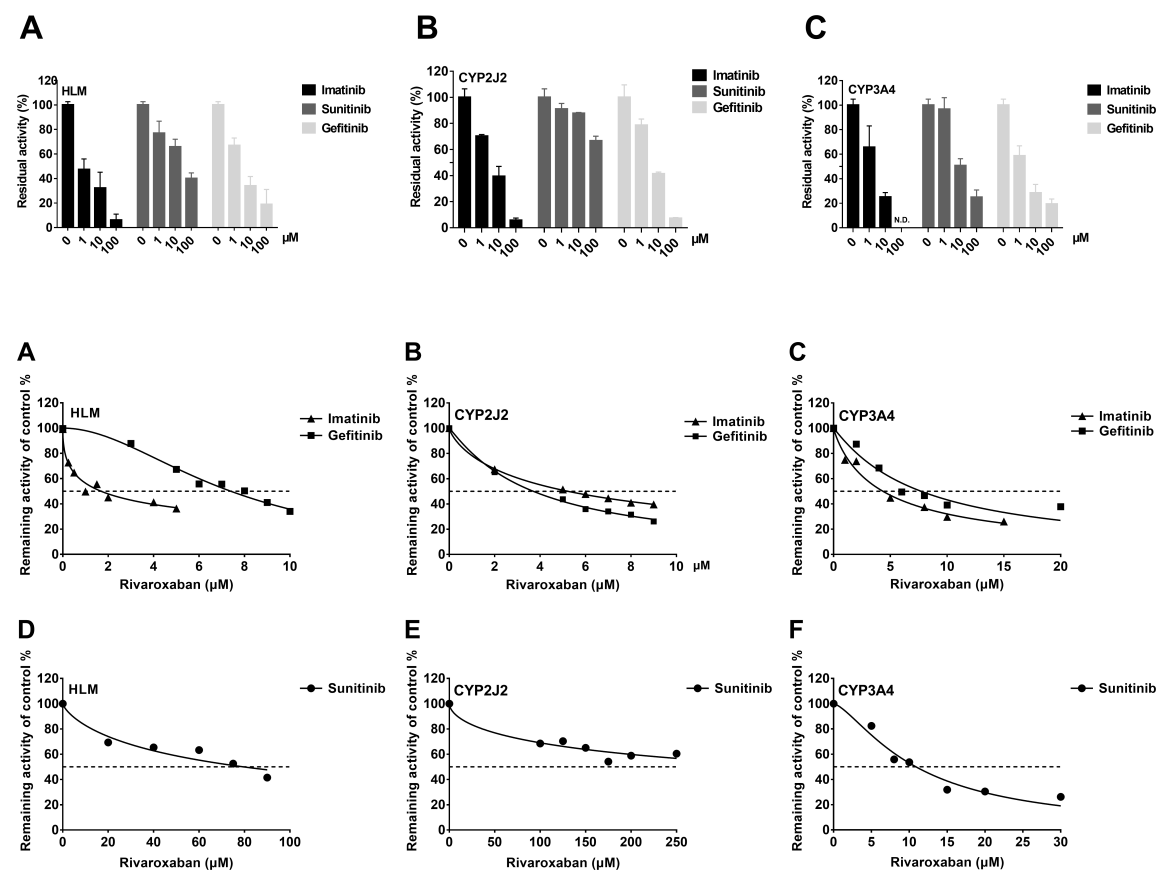

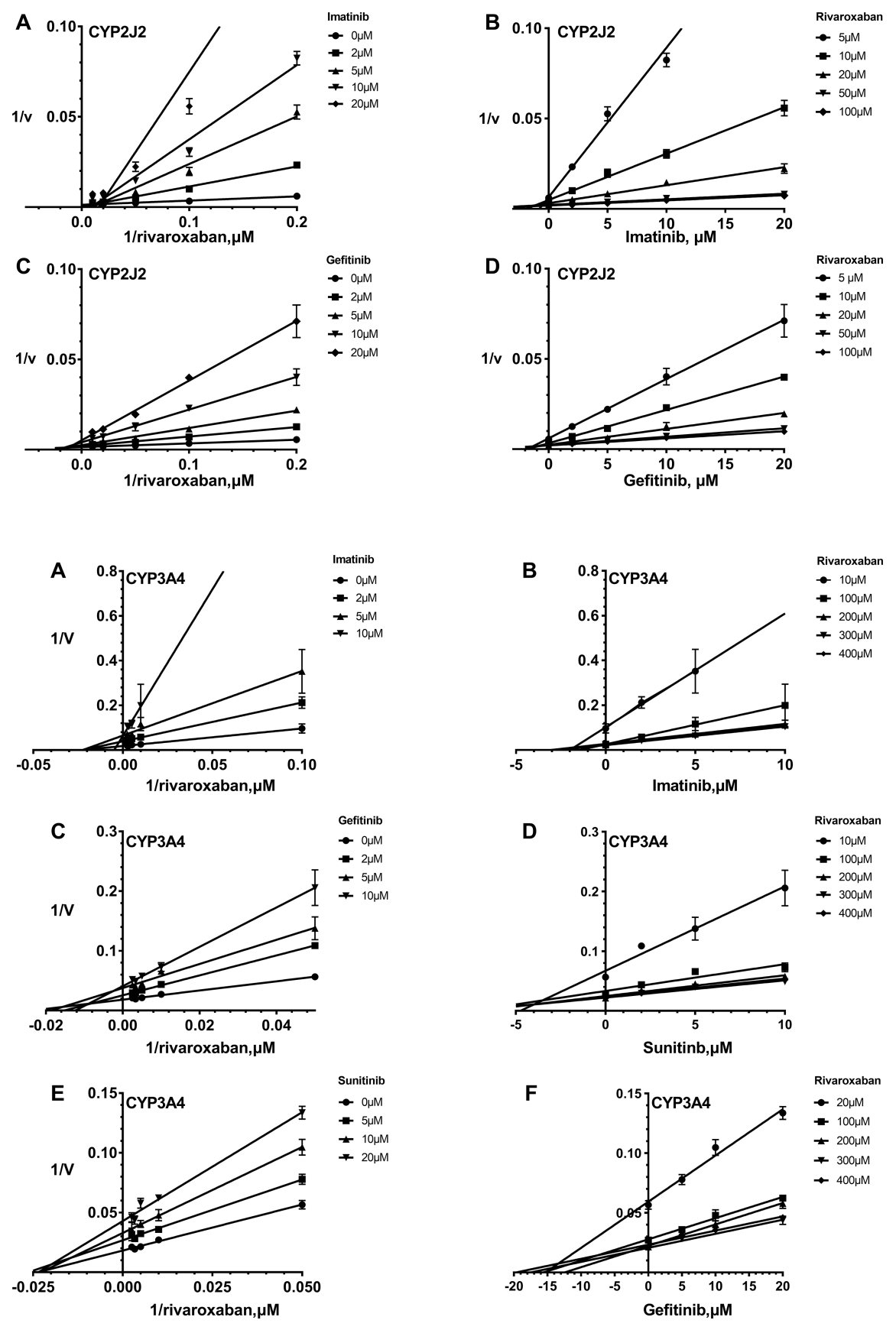

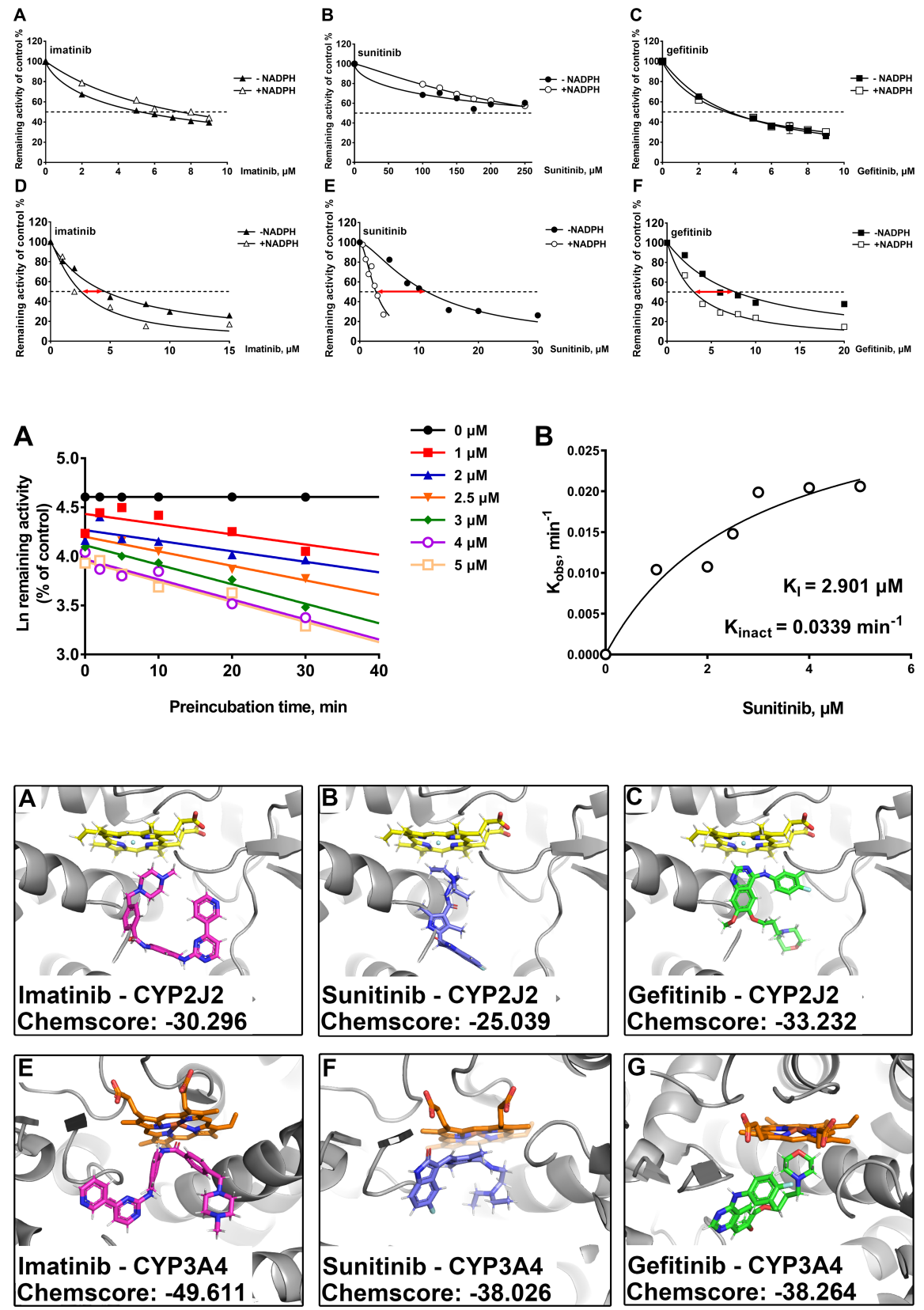